\title{
The aims and activities of the International Network of Nuclear Structure and Decay Data Evaluators
}

\author{
A.L. Nichols ${ }^{1, a}$ and J.K. Tuli ${ }^{2}$ \\ ${ }^{1}$ Nuclear Data Section, Division of Physical and Chemical Sciences, Department of Nuclear Sciences and Applications, \\ International Atomic Energy Agency, Wagramer Strasse 5, 1400 Vienna, Austria \\ 2 National Nuclear Data Center, Brookhaven National Laboratory, Upton, NY 11973-5000, USA
}

\begin{abstract}
The International Network of Nuclear Structure and Decay Data (NSDD) Evaluators consists of a number of evaluation groups and data service centres in several countries that appreciate the merits of working together to maintain and ensure the quality and comprehensive content of the ENSDF database (Evaluated Nuclear $\underline{\text { Structure }}$

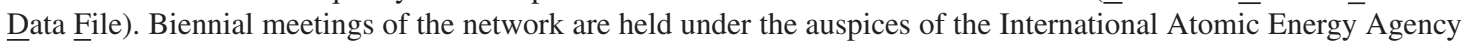
(IAEA) to assign evaluation responsibilities, monitor progress, discuss improvements and emerging difficulties, and agree on actions to be undertaken by individual members. The evaluated data and bibliographic details are made available to users via various media, such as the journals Nuclear Physics A and Nuclear Data Sheets, the World Wide Web, on CD-ROM, wall charts of the nuclides and Nuclear Wallet Cards. While the ENSDF master database is maintained by the US National Nuclear Data Center at the Brookhaven National Laboratory, these data are also available from other nuclear data centres including the IAEA Nuclear Data Section. The Abdus Salam International Centre for Theoretical Physics (ICTP), Trieste, Italy, in cooperation with the IAEA, organizes workshops on NSDD at regular intervals. The primary aims of these particular workshops are to provide hands-on training in the data evaluation processes, and to encourage new evaluators to participate in NSDD activities. The technical contents of these NSDD workshops are described, along with the rationale for the inclusion of various topics.
\end{abstract}

\section{Introduction}

Reliable nuclear structure and decay data represent the fundamental backbone of nuclear physics and astrophysics research, and are also of vital importance in a significant number of applied nuclear fields such as power generation and associated fuel cycle operations (e.g., fuel manufacture, transport, reprocessing and waste management), materials analysis, medical diagnosis and radiotherapy. There is a continuous demand for recommended good quality data formulated through the speedy incorporation of new and improved measurements. Nuclear data are incorporated into the Evaluated $\underline{\text { Nuclear }}$ Structure Data File (ENSDF) as:

(a) decay data - including half-lives, emission energies and probabilities for transition types that cover $\alpha, \beta^{-}, \mathrm{EC}$, $\mathrm{EC}+\beta^{+}, \mathrm{IT}, \gamma, \beta^{-} \mathrm{n}$ and $\mathrm{p}$ decay,

(b) reaction data - relevant nuclear structure data from various nuclear reactions,

(c) adopted data - recommended nuclear level properties derived by critical analyses of all available experimental information from decay and reaction studies,

that are ordered in terms of over 290 isobaric chains for convenience and speedy assessment. Comprehensive, upto-date and well-ordered databases are an essential prerequisite for the nuclear physics research community and

${ }^{a}$ Presenting author, e-mail: a.nichols@iaea.org applications specialists who require credible and reliable data at the press of a key. ENSDF is a crucial database in such work [1-4].

Although various theoretical models predict the existence of over 6000 radionuclides, many are difficult to produce in a laboratory environment - experimental information has been generated for nearly 3000 of these nuclides, while more than 3000 remain undiscovered. This area of nuclear physics research is an extremely challenging frontier that can greatly benefit from systematic studies of the accumulated knowledge of 100 years of nuclear structure studies.

Nuclear structure and decay data are compiled and evaluated by a collaborating team of specialists that have met at regular intervals since 1974 as the International Network of Nuclear Structure and Decay Data Evaluators under the auspices of the International Atomic Energy Agency (IAEA). The ENSDF master database is maintained by staff of the US National Nuclear Data Center at Brookhaven National Laboratory, Upton, New York, USA. Several countries such as Belgium, Canada, China, France, Japan, Kuwait, the Russian Federation and the United States of America have contributed over many years. New evaluation teams have recently formed in other countries to increase the international flavour of this initiative, including Argentina, Australia, Brazil, Bulgaria and India. However, despite this array of multinational cooperative effort, the on-going commitment effectively totals only 9.5 full-time scientists per annum for an effort that is estimated to require the continuous input of 12 full-time scientists per annum to maintain the desired currency and quality of ENSDF. 


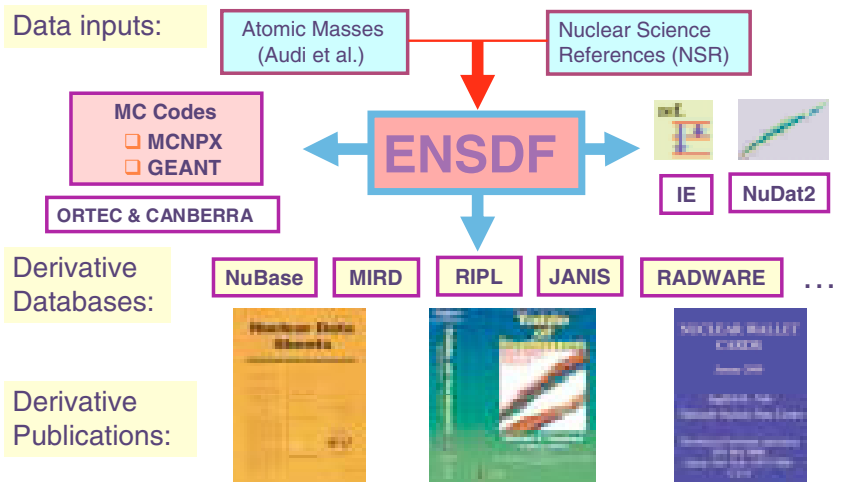

Fig. 1. ENSDF: major data sources and derivatives.

\section{International Network of Nuclear Structure and Decay Data Evaluators}

Nuclear structure and decay data (NSDD) compilations and evaluations have an impressive history that stretches back to the 1930s, with the publication of the first known tabulations of recommended nuclear parameters by Curie et al. [5] and Fea [6]. These tabulations were followed in 1940 by what was essentially to become the popular Table of Isotopes as conceived originally by Livingood and Seaborg [7]. Clearly, this type of extremely useful work has developed and expanded considerably since those times, despite an early statement that "the rate at which radioactivities are discovered may be reduced very considerably and the table would itself become stable". These and other parallel evaluation efforts continued, and the familiar presentational style of Nuclear Data Sheets appeared in early 1966 [8]. ENSDF represents the electronic nuclear structure and decay data file that constitutes the primary input for Nuclear Data Sheets, Table of Isotopes, Nuclear Wallet Cards, and other products familiar to the nuclear physics and data communities (fig. 1).

\subsection{Mass chain evaluations}

Since the beginning of May 1974, evaluators working on ENSDF have held collaborative meetings every two years to agree and assign mass chain evaluations, monitor progress, and discuss improvements and emerging difficulties. This network began at a time when the workload was heavily reliant upon American input. A more equitable involvement of other national laboratories was envisaged and partially achieved.

Network meetings are sponsored by the IAEA Nuclear Data Section, and have the following primary objectives:

(a) coordination of the work of all evaluation centres and groups participating in the compilation, evaluation and dissemination of NSDD,

(b) maintenance and improvement of the rules and standards governing NSDD evaluations,

(c) regular review of the development and use of computerized systems and databases maintained specifically for NSDD activities.

The $16^{\text {th }}$ meeting of the network took place at McMaster University, Hamilton, Canada, from 6 to 10 June 2005 [9], and plans are underway to hold the $17^{\text {th }}$ meeting at Petersburg Nuclear Physics Institute, St. Petersburg, Russian Federation, on 11 to 15 June 2007. Under normal circumstances, the first two days of such gatherings are dedicated to a combination of technical reviews and discussion papers, addressing particular topics in which progress has been made and problems have been experienced over the previous 24 months. Various software packages are regularly being developed to assist the mass chain evaluators in their work, and future plans in this area can be aired at this time. Specific mass chain activities and administrative issues are debated over the final three days. Network contacts, affiliations and their mass chain responsibilities are listed in table 1.

\subsection{Other nuclear properties}

Other specific nuclear properties are comprehensively compiled and evaluated by individual members of the network without limiting their efforts to a particular mass chain. These horizontal evaluations include atomic masses [10], nuclear magnetic and electric moments [11], electric monopole strengths [12], capture gamma rays [13] and various other nuclear parameters. An additional database entitled XUNDL is also maintained primarily by the McMaster group that provides a rapid means of Internet access to the most recent publications and pre-prints of experimental nuclear structure data that are not yet available in the ENSDF database [14].

\subsection{Manpower}

There are many distinct advantages associated with maintaining a healthy database by means of a multinational network:

(a) maintenance of a well-defined archive of nuclear structure and decay data for future generations,

(b) healthy interplay between nuclear reaction and decay data to define nuclear parameters,

(c) constructive impact of recommended nuclear structure data on developing nuclear theories,

(d) assistance in the resolution of contradictory results,

(e) identification of requirements for and stimulation of new measurements,

(f) benefits to users in many applied areas, including nuclear medicine, analytical science, environmental monitoring, nuclear engineering, etc.

Members of the multinational NSDD network can take great personal pride in assisting such an impressive array of applied and pure nuclear physicists with their many and varied problems and nuclear data needs.

Nevertheless, the organisers of the international NSDD network have become aware in recent years of an increasing problem in maintaining and updating mass chain evaluations for ENSDF with the necessary regularity. Evidence of a shortfall in effort has been detected over the previous ten years, as evaluators in Europe have retired without any obvious replacements (table 2). While some progress has been made in recruitment through the commitment of nuclear physics institutes in China and India for this essential work, these welcome additions are not fully commensurate with the losses 
Table 1. International Network of Nuclear Structure and Decay Data Evaluators (2005/06), and co-workers.

\begin{tabular}{|c|c|c|}
\hline $\begin{array}{l}\text { Country/ } \\
\text { affiliation }\end{array}$ & $\begin{array}{l}\text { Contact and } \\
\text { co-workers }\end{array}$ & $\begin{array}{l}\text { Assigned } \\
\text { mass } \\
\text { chains }\end{array}$ \\
\hline $\begin{array}{l}\text { Argentina } \\
\text { CNEA, Buenos Aires }\end{array}$ & G.V. Marti & $\begin{array}{l}(178,191, \\
193)\end{array}$ \\
\hline $\begin{array}{l}\text { Australia } \\
\text { ANU, Canberra }\end{array}$ & T. Kibédi & $172-175$ \\
\hline $\begin{array}{l}\text { Belgium } \\
\text { Universiteit Gent }\end{array}$ & D.J.A. De Frenne & $\begin{array}{l}102,103 \\
105,106, \\
110,112\end{array}$ \\
\hline $\begin{array}{l}\text { Brazil } \\
\text { U. de São Paulo }\end{array}$ & V.R. Vanin & $(191,193)$ \\
\hline $\begin{array}{l}\text { Bulgaria } \\
\text { University of Sofia }\end{array}$ & $\begin{array}{l}\text { D.L. Balabanski } \\
\text { S.P. Lalkovski }\end{array}$ & $(112,200)$ \\
\hline $\begin{array}{l}\text { Canada } \\
\text { McMaster University, } \\
\text { Hamilton }\end{array}$ & $\begin{array}{l}\text { B. Singh } \\
\text { J.A. Cameron }\end{array}$ & $\begin{array}{l}1,31-44, \\
64,89,98, \\
100,149, \\
151,164, \\
188,190, \\
194\end{array}$ \\
\hline $\begin{array}{l}\text { China } \\
\text { CNDC, CIAE, } \\
\text { Beijing } \\
\text { Jilin University, } \\
\text { Changchun } \\
\end{array}$ & $\begin{array}{l}\text { Ge Zhigang } \\
\text { Huang Xiaolong } \\
\text { Huo Junde }\end{array}$ & $\begin{array}{l}51-56 \\
62,63 \\
195-198\end{array}$ \\
\hline $\begin{array}{l}\text { France } \\
\text { CEA Bruyères-le-Châtel }\end{array}$ & J. Blachot & $\begin{array}{l}101,104, \\
107-109 \\
111 \\
113-117\end{array}$ \\
\hline $\begin{array}{l}\text { India } \\
\text { IIT, Roorkee } \\
\text { Manipal University }\end{array}$ & $\begin{array}{l}\text { A.K. Jain } \\
\text { M. Gupta }\end{array}$ & $218-229$ \\
\hline $\begin{array}{l}\text { Japan } \\
\text { JAEA, Ibaraki-ken }\end{array}$ & $\begin{array}{l}\text { J. Katakura } \\
\text { T. Tamura }\end{array}$ & $118-129$ \\
\hline $\begin{array}{l}\text { Kuwait } \\
\text { University of Kuwait }\end{array}$ & A.R. Farhan & $74-80$ \\
\hline $\begin{array}{l}\text { Russian Federation } \\
\text { PNPI, St. Petersburg }\end{array}$ & $\begin{array}{l}\text { I.A. Mitropolsky } \\
\text { A. Rodionov } \\
\text { Yu. Khazov }\end{array}$ & $130-135$ \\
\hline $\begin{array}{l}\text { United Kingdom } \\
\text { University of Oxford }\end{array}$ & N.J. Stone & - \\
\hline
\end{tabular}

experienced in Europe, a region of the world that might have been expected to ensure some re-generation of expertise in this vital area of research and development.

\section{Workshops on Nuclear Structure and Decay Data: Theory and Evaluation, ICTP, Trieste, Italy}

The Abdus Salam International Centre for Theoretical Physics (ICTP, Trieste, Italy) in cooperation with the International
Table 1 . (cont'd.)

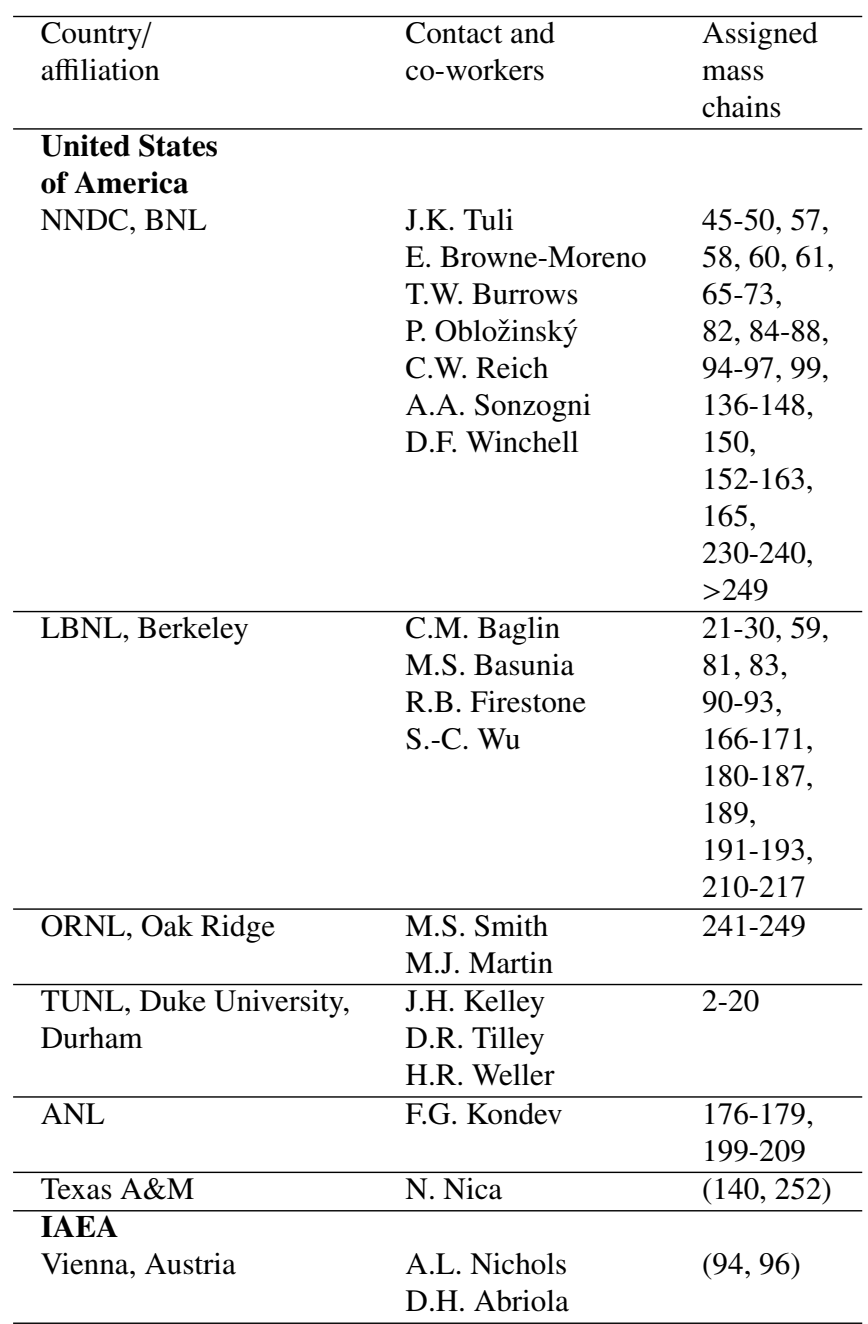

( ) denotes partially shared responsibility.

Atomic Energy Agency has organised workshops on Nuclear Structure and Decay Data: Theory and Evaluation on a regular basis since November 2003. These workshops have been held at Abdus Salam ICTP, and constitute a unique opportunity for scientists to gain extensive and up-to-date training on the evaluation of nuclear structure and decay data as developed for the preparation of ENSDF files. Nuclear physicists from all countries that are members of the United Nations, UNESCO or the IAEA may attend such workshops. While the main purpose of such ICTP initiatives is to assist scientists from developing nations through a programme of training activities, applicants from developed countries are also welcome to attend.

A one-week pilot workshop was held at IAEA Headquarters from 18 to 22 November 2002 [15] to assist in the evolving design and development of the subsequent two-week workshops at ICTP. Eight participants were selected in consultation with the International Network of NSDD Evaluators to ensure they possessed an appropriate level of experience in nuclear structure and decay data studies, and the potential to become fully fledged mass chain evaluators. Under these circumstances, a particularly successful five days resulted in the rapid 
Table 2. Number of member laboratories/institutes responsible for ENSDF mass chain evaluations.

\begin{tabular}{lcccc}
\hline & \multicolumn{4}{c}{ Year } \\
& 1981 & 1986 & 1996 & 2007 \\
\hline N. America & 6 & 6 & 6 & 6 \\
Europe & 6 & 5 & 4 & $2 \rightarrow 1(0)$ \\
Russia & 2 & 2 & 2 & 1 \\
Japan & 1 & 1 & 1 & 1 \\
China & - & - & 1 & 2 \\
Rest of the World & 1 & 1 & 1 & 3 \\
\hline & 16 & 15 & 15 & $14(13)$ \\
\hline
\end{tabular}

introduction of Marti (Argentina), Kibédi (Australia), Vanin (Brazil), Balabanski (Bulgaria), Huang Xiaolong (China), Jain (India), Mitropolsky (Russian Federation) and Kondev (ANL, USA) into the evaluators' network (table 1).

A number of important lessons were learnt from the pilot study that assisted greatly in the evolution of the following two-week workshops at ICTP:

(1) 17 to 28 November 2003, ICTP, Trieste, Italy; 24 participants and 11 lecturers/demonstrators [16].

(2) 4 to 15 April 2005, ICTP, Trieste, Italy; 27 participants and 11 lecturers/demonstrators [17].

(3) 20 February to 3 March 2006, ICTP Trieste, Italy; 23 participants and 11 lecturers/demonstrators [18].

The computer-based laboratory facilities at ICTP are superior to those available at IAEA Headquarters, and were admirably suited to the implementation of individual exercises by approximately 30 students under a central control system as required for the ENSDF model exercises (see below).

\subsection{Content}

The freshness of the workshop has been maintained through deliberate changes to their detailed content over the four years they have been staged, although the primary technical objectives have remained identified with the following topics:

(a) modern nuclear models,

(b) relevant experimental techniques,

(c) statistical analyses to derive recommended data,

(d) evaluation methodologies for nuclear structure and decay data,

(e) international efforts to produce ENSDF.

The first two fundamental topics cover the basic tenets of any advances in nuclear physics, while the latter three areas of expertise constitute the means of generating reputable recommended nuclear data in forms of immediate value to the scientific community. All facets of the workshop are strongly interlinked, and are suitably arranged and presented in ways that maintain the students' attention and interest.

Extensive presentations of the most pertinent scientific material are given by invited lecturers, along with the introduction of computer-based exercises and workshop tasks.
Participants are also invited to contribute their own thoughts and presentations of direct relevance to the workshop. Furthermore, students are given the opportunity to review the contents of the programme through a written questionnaire and direct discussions close to the end of the two weeks of work.

Significant quantities of written material and various Powerpoint presentations have been prepared. Therefore, a relatively large fraction of this knowledge has been assembled in the form of working manuals for distribution and use at future workshops (see refs. [16-18] and Web page www-nds.iaea.org/workshops/ictp2006/). Lecture topics have included the following:

(a) Nuclear theory

- nuclear shell and interacting boson models, P. Van Isacker (GANIL, France),

- single particle and collective models, P. Van Isacker (GANIL, France),

- geometrical symmetries in nuclei, A.K. Jain (IIT, Roorkee, India),

- Hartree-Fock-Bogoliubov method, D. Vretenar (University of Zagreb, Croatia),

- self-consistent mean-field models, D. Vretenar (University of Zagreb, Croatia),

- structure of odd-even nuclei in the interacting boson model, S. Brant (University of Zagreb, Croatia),

- high spin states in the interacting boson and bosonfermion models, S. Brant (University of Zagreb, Croatia),

- structure of odd-odd nuclei in the interacting bosonfermion model, S. Brant (University of Zagreb, Croatia),

- $\beta^{-}$decay, S. Brant (University of Zagreb, Croatia),

- quasiparticle or BCS method, Y.K. Gambhir (IIT, Mumbai, India),

- Hartree-Fock mean field theory, Y.K. Gambhir (IIT, Mumbai, India).

(b) Experimental nuclear spectroscopy

- nuclear shapes, lifetimes, P. von Brentano (IKUK, Cologne, Germany),

- nuclear structure physics, F.G. Kondev (ANL, USA),

- deduction of $\mathbf{J}^{\pi}$, T. Kibédi (ANU, Australia),

- characterizing nuclei using separators, T. Kibédi (ANU, Australia).

(c) Statistical analyses

- evaluation of discrepant data, T.D. MacMahon (NPL, UK).

\section{(d) ENSDF}

- introduction and history, J.K. Tuli (BNL, USA),

- bibliographic databases and utility codes, T.W. Burrows (BNL, USA),

- decay data, E. Browne-Moreno (LBNL, USA),

- reaction data, C.M. Baglin (LBNL, USA),

- adopted levels and gammas, C.M. Baglin (LBNL, USA),

- model exercises,

- ENSDF evaluation manuals. 
(e) Additional material

- relevant IAEA coordinated research projects and other related activities, A.L. Nichols (IAEA, Austria),

- International Network of Nuclear Structure and Decay Data Evaluators, A.L. Nichols (IAEA, Austria),

- Web pages, T.W. Burrows et al.

This wealth of information is freely available on request from: services@iaeand.iaea.org.

Workshop participants are introduced to mass chain evaluations through group and individual PC activities that represent $50 \%$ of the work undertaken during the second week. A further noteworthy point is that specific participants exhibiting the greatest interest and promise were identified for possible future involvement in NSDD and mass chain evaluations. If appropriate, agreed mentoring processes were subsequently established between such individuals, their research institutes and a willing member of the NSDD network with the aim of encouraging their longer-term involvement in such important work.

\subsection{Participants}

A summary of the country origins of past attendees at the IAEA and ICTP workshops is given in table 3. Such events have a strong educational function, but may also serve to identify those students who may have sufficient personal interest to consider undertaking their own mass chain evaluations in the future.

Efforts will continue to develop the contents of further workshops to the benefit of the participants and the International Network of Nuclear Structure and Decay Data Evaluators. Hopefully, a two-week workshop of this type can be held at Abdus Salam International Centre for Theoretical Physics on a regular basis every two or three years.

\section{Future requirements}

Popular use and adoption of data within any electronic database is largely determined by the following:

(a) reliability and credibility - data must be correctly evaluated and incorporated into the database,

(b) comprehensive - database should include all measured quantities and their uncertainties,

(c) up-to-date - results from all measurements should be promptly incorporated into the database,

(d) accessibility - easy and rapid availability.

The International Network of Nuclear Structure and Decay Data Evaluators aims to achieve these objectives to the best of the members' abilities.

An important point of note in any nuclear data compilation involves the significant amount of data that is never published in the public domain due to a lack of space within journal publications. A dedicated repository for such important support data has been proposed in which nuclear physicists can place such material and provide all additional details of their work. However, this concept requires the full cooperation of the
Table 3. Attendance at IAEA and ICTP workshops on nuclear structure and decay data.

\begin{tabular}{llll}
\hline Country & No. & Country & No. \\
\hline Algeria & 3 & Mongolia & 2 \\
Argentina & $3(3)$ & Nigeria & 1 \\
Australia & $1(1)$ & Pakistan & 1 \\
Bangladesh & 3 & Romania & $4(1)$ \\
Brazil & $4(2)$ & Russian & $5(2)$ \\
Bulgaria & $3(2)$ & Federation & \\
& & Spain & 1 \\
Cameroon & 3 & Sudan & 2 \\
China & $8(2)$ & Syria & 1 \\
Egypt & 3 & Turkey & 1 \\
France & 2 & UK & 2 \\
Hungary & 1 & USA & $2(2)$ \\
& & Ukraine & 1 \\
India & $15(6)$ & Uzbekistan & 1 \\
Iran & 5 & & 1 \\
Iraq & 1 & Vietnam & \\
Republic of & 2 & & \\
Korea & & & \\
\hline
\end{tabular}

() denotes number of subsequent mass chain evaluators, albeit sometimes only temporary.

administrative editors of the existing journals of importance in nuclear science, and this has proved difficult to achieve in an appropriate and speedy manner.

The survival and maintenance of the quality of ENSDF depends on the recruitment of new data evaluators to replace the ageing nuclear physicists undertaking this work. Unless new blood can be introduced soon, there is a serious danger that the current loose confederation of dedicated participants will fade away - there is an urgent need for younger scientists to join the NSDD evaluation network. An initiative was launched by the IAEA in 2002/03 to establish regular workshops at ICTP, Trieste, Italy, for the training of new nuclear structure evaluators. The IAEA also provides seed-funding to ensure that the most promising students are able to contribute to the mass chain evaluations beyond the workshops by means of an agreed mentoring process that involves individual members of the NSDD network. There can be no doubt that the assistance of the worldwide nuclear physics research community is urgently required to ensure the survival of ENSDF at the necessary level of credibility, reliability and quality.

The authors wish to express their gratitude to all colleagues within the International Network of NSDD Evaluators for their enthusiastic efforts to maintain the quality of the existing nuclear structure database. Work undertaken by J.K. Tuli is supported by the US Department of Energy, Office of Nuclear Physics, Office of Science, under contract No. DE-AC02-98CH10886. 


\section{References}

1. ENSDF, National Nuclear Data Center, Brookhaven National Laboratory, Upton, New York, USA-Web page www.nndc.bnl.gov; Nuclear Data Section, International Atomic Energy Agency, Vienna, Austria-Web page www-nds.iaea.org.

2. Nuclear Physics A (Elsevier, Amsterdam, The Netherlands).

3. Nuclear Data Sheets (Elsevier, Amsterdam, The Netherlands).

4. A.A. Sonzogni, J.K Tuli, Nuclear structure and radioactive decay resources at the US National Nuclear Data Center, Int. Conf. on Nuclear Data for Science and Technology (ND2007), Nice, France, April 2007 (these proceedings).

5. M. Curie, A. Debierne, A.S. Eve, H. Geiger, O. Hahn, S.C. Lind, St. Meyer, E. Rutherford, E. Schweidler, The radioactive constants as of 1930, Rev. Mod. Phys. 3, 427 (1931).

6. G. Fea, Tabelle riassuntive e bibliografia delle transmutazioni artificiali, Nuovo Cimento 12, 368 (1935).

7. J.J. Livingood, G.T. Seaborg, A table of induced radioactivities, Rev. Mod. Phys. 12, 30 (1940).

8. Nuclear Data Sheets, Section B, Vol. 1, Issue 1, February 1966 (Academic Press Inc., New York and London).

9. A.L. Nichols, J.K. Tuli, Summary Report of an IAEA Technical Meeting on Coordination of the International Network of Nuclear Structure and Decay Data Evaluators, 6-10 June 2005, McMaster University, Hamilton, Canada, IAEA Report INDC(NDS)-0476, September 2005.

10. A.H. Wapstra, G. Audi, C. Thibault, The AME2003 atomic mass evaluation, Nucl. Phys. A 729, 129 (2003).

11. N.J. Stone, Table of nuclear magnetic dipole and electric quadrupole moments, At. Data Nucl. Data Tables 90, 75 (2005).
12. T. Kibédi, R.H. Spear, Electric monopole transitions between $\mathrm{O}^{+}$states for nuclei throughout the periodic table, At. Data Nucl. Data Tables 89, 77 (2005).

13. H.D. Choi, R.B. Firestone, R.M. Lindstrom, G.L. Molnár, S.F. Mughabghab, R. Paviotti-Corcuera, Z. Révay, A. Trkov, V. Zerkin, Chunmei Zhou, Database of prompt gamma rays from slow neutron capture for elemental analysis, International Atomic Energy Agency, Vienna, Austria (2007), ISBN 92-0101306-X.

14. XUNDL is accessible on nds121.iaea.org/endsf/xunindex.jsp and www.nndc.bnl.gov/endsf/xunindex.jsp.

15. V.G. Pronyaev, A.L. Nichols, Summary Report of Workshop on Nuclear Structure and Decay Data Evaluation, 18-22 November 2002, IAEA Headquarters, IAEA report INDC(NDS)-439, January 2003; also available on www-nds.iaea.org/reports-new/indcreports/indc-nds/.

16. A.L. Nichols, P.K. McLaughlin, Workshop on Nuclear Structure and Decay Data: Theory and Evaluation - Manual, Parts 1 and 2, 17-28 November 2003, ICTP, Trieste, Italy, IAEA report INDC(NDS)-452, January 2004; also available on wwwnds.iaea.org/reports-new/indc-reports/indc-nds/.

17. A.L. Nichols, P.K. McLaughlin, Workshop on Nuclear Structure and Decay Data: Theory and Evaluation, Addendum-2005, 4 - 15 April 2005, ICTP, Trieste, Italy, IAEA Report INDC (NDS)-0473, July 2005; also available on www-nds.iaea.org/ reports-new/indc-reports/indc-nds/.

18. A.L. Nichols, P.K. McLaughlin, Workshop on Nuclear Structure and Decay Data: Theory and Evaluation, Addendum2006, 20 February-3 March 2006, ICTP, Trieste, Italy, IAEA report INDC(NDS)-0496, June 2006; also available on wwwnds.iaea.org/reports-new/indc-reports/indc-nds/. 